\title{
Análise epidemiológica e espacial da COVID-19 no Estado do Piauí
}

\author{
Epidemiological and spatial analysis of COVID-19 in the State of Piauí \\ Análisis epidemiológico y espacial de COVID-19 en el Estado de Piauí
}

Recebido: 21/04/2021 | Revisado: 30/04/2021 | Aceito: 03/05/2021 | Publicado: 16/05/2021

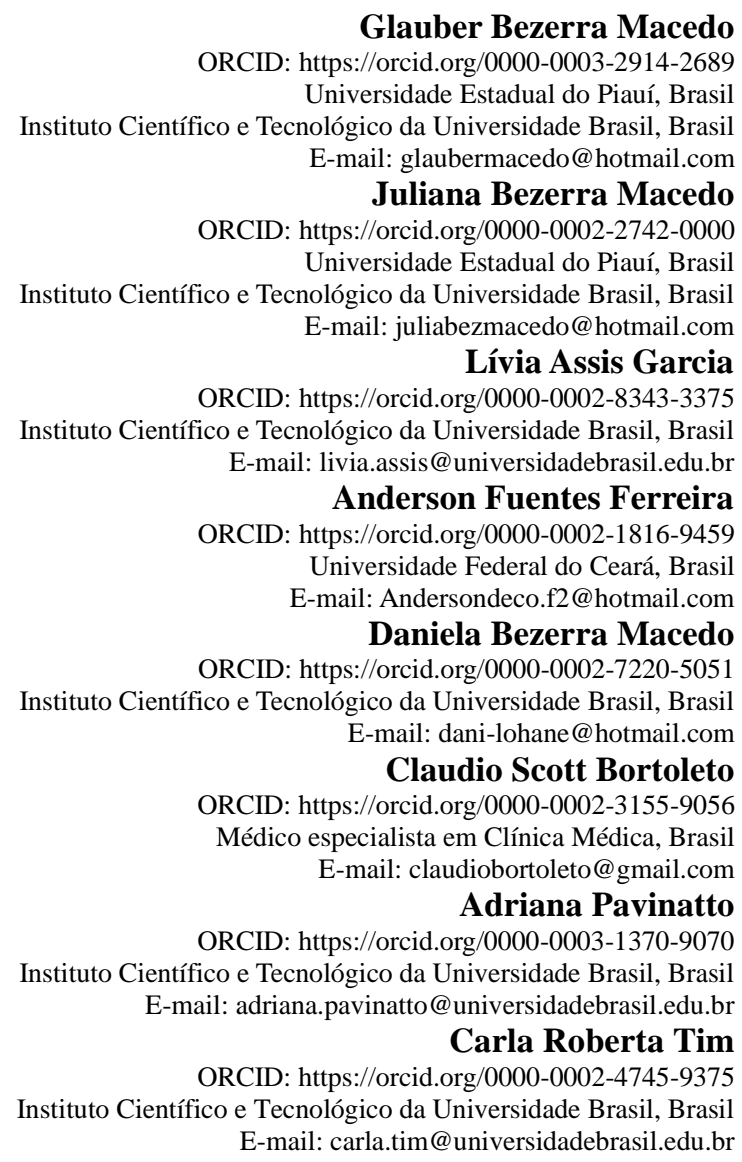

\section{Resumo}

Estudo ecológico, analítico e exploratório que objetivou realizar análises epidemiológicas e espaciais da distribuição dos casos confirmados e dos óbitos por doença do Coronavírus 2019 (do inglês, Coronavírus Disease 2019 - COVID19) que ocorreram no ano de 2020, no estado do Piauí, Brasil. As variáveis elencadas foram obtidas via banco de dados do IBGE e SESAPI. Nas análises epidemiológicas foram realizadas estatísticas descritivas e nas análises espaciais, foram construídos mapas utilizando técnicas de geoprocessamento, através das análises estatísticas de Moran Global e Local. Os resultados evidenciaram que, no ano de 2020, foram notificados 143.179 casos confirmados e 2.240 óbitos causados por COVID-19 no Piauí. Observou-se maior percentual de casos em indivíduos do sexo feminino, adultos jovens, e os óbitos em idosos, do sexo masculino, com doenças crônicas. Houve formação de clusters no estado, elementos a serem considerados na organização e fortalecimento dos serviços de saúde para o desenvolvimento de estratégias que busquem minimizar a transmissão durante a emergência da COVID-19.

Palavras-chave: COVID-19; Coronavírus; Epidemiologia; Análise espacial.

\begin{abstract}
Ecological, analytical and exploratory study that aimed to carry out epidemiological and spatial analyzes of the distribution of confirmed cases and deaths from Coronavirus 2019 disease (from English, Coronavirus Disease 2019 COVID-19) that occurred in 2020, in the state of Piauí, Brazil. The variables listed were obtained via the IBGE and SESAPI database. In the epidemiological analyzes, descriptive statistics were performed and in the spatial analyzes, maps were constructed using geoprocessing techniques, through the statistical analyzes of Moran Global and Local. The results showed that, in 2020,143,179 confirmed cases and 2,240 deaths caused by COVID-19 were reported in Piauí. There was a higher percentage of cases in females, young adults, and deaths in the elderly, males, with chronic
\end{abstract}


diseases. Clusters were formed in the state, elements to be considered in the organization and strengthening of health services for the development of strategies that seek to minimize transmission during the emergence of COVID- 19 .

Keywords: COVID-19; Coronavirus; Epidemiology; Spatial analysis.

\begin{abstract}
Resumen
Estudio ecológico, analítico y exploratorio que tuvo como objetivo realizar análisis epidemiológicos y espaciales de la distribución de casos confirmados y defunciones por enfermedad Coronavirus 2019 (del inglés, Enfermedad Coronavirus 2019 - COVID-19) ocurridos en 2020, en el estado de Piauí, Brasil. Las variables enumeradas se obtuvieron a través de la base de datos IBGE y SESAPI. En los análisis epidemiológicos se realizó estadística descriptiva y en los análisis espaciales se construyeron mapas utilizando técnicas de geoprocesamiento, a través de los análisis estadísticos de Moran Global y Local. Los resultados mostraron que, en 2020, se reportaron 143.179 casos confirmados y 2.240 muertes por COVID-19 en Piauí. Hubo un mayor porcentaje de casos en mujeres, adultos jóvenes y muertes en ancianos, hombres, con enfermedades crónicas. Se conformaron clusters en el estado, elementos a considerar en la organización y fortalecimiento de los servicios de salud para el desarrollo de estrategias que busquen minimizar la transmisión durante el surgimiento de COVID-19.
\end{abstract}

Palabras clave: COVID-19; Coronavirus; Epidemiología; Análisis espacial.

\title{
1. Introdução
}

Coronavírus $(\mathrm{CoV})$ é um grupo de vírus que causam doenças envolvendo os sistemas respiratórios, entérico, hepático e neurológico, com gravidade variável. A maioria dos coronavírus causa doença em animais. No entanto, sete tipos de CoV são conhecidos por causar doença em seres humanos (HCoV-229E, HCoV-OC43, HCoV-NL63, HCoV-HKU1, SARS-CoV, MERS-CoV e SARS-CoV-2) (Cheng \& Shan, 2020). Estes CoV, tradicionalmente, causavam uma baixa porcentagem de infeções respiratórias anuais. Entretanto, nos últimos 20 anos, com a identificação do SARS-CoV e do MERS-CoV, denominadas de síndrome respiratória aguda grave e síndrome respiratória do Oriente Médio, respectivamente, e mais recentemente o novo Coronavírus, SARS-CoV-2, objeto deste estudo, ocorreram o aumento do número das infecções por CoV (Zumla et al., 2016; Channappanavar et al., 2014; Cheng et al., 2007).

O SARS-CoV-2, foi identificado por meio de casos de uma síndrome respiratória em Wuhan, na China, em dezembro de 2019, despertando atenção mundial devido sua rápida disseminação global (Huang et al., 2020; Thompson, 2020; Heymann \& Shindo, 2020). A doença, que foi denominada Coronavírus Disease 2019 (COVID-19) causa uma síndrome respiratória aguda grave que ocasiona uma lesão pulmonar aguda (LPA) e a síndrome do desconforto respiratório agudo (SDRA), uma das complicações crítica da infecção (Gorbalênya et al., 2020; Zhu et al., 2020).

A COVID-19 se disseminou por todos os continentes e foi declarada pela Organização Mundial da Saúde (OMS) como um caso de emergência de saúde pública de interesse internacional (WHO, 2020a). Até 31 de dezembro de 2020, o mundo registrou 83.522.000 de casos confirmados e 1.826.000 de mortes (WHO, 2020b).

Suas características epidemiológicas ainda não estão totalmente determinadas, mas sabe-se que se trata de um vírus infeccioso, com alta prevalência e ampla distribuição (Lipsitch et al., 2020). Sua transmissão se dá por meio da disseminação de partículas virais, provenientes do trato respiratório superior de indivíduos contaminados, através do ar, água e superfícies contaminadas (Lai et al., 2020; Van et al., 2020). O vírus pode permanecer viável de hora a dias, a depender do ambiente, material e fatores físico-químicos, como temperatura e pH, onde se encontra (Kempf et al., 2020). Por se tratar de uma doença altamente transmissível, as análises dos dados são fundamentais para conhecimento da situação epidemiológica local (dos Santos \& Mendes, 2020).

As estatísticas evidenciam o aumento progressivo dos casos de COVID-19 e através de análises espaciais, método este muito utilizado em saúde coletiva, é possível detectar aglomerados da doença, o que contribui para o diagnóstico da situacional e planejamento de ações pelos serviços de saúde (Kang et al., 2020). Em se tratando de uma doença pandêmica, considera-se a 
importância dessa ferramenta como suporte para implementação de medidas de controle (Belforte et al., 2020; Cardoso et al., 2020).

Nesse contexto, o presente estudo teve como objetivo caracterizar e analisar distribuição espacial dos casos diagnosticados e dos óbitos ocorridos por COVID-19, no ano de 2020, no estado do Piauí, Brasil.

\section{Metodologia}

Essa pesquisa trata-se de um estudo ecológico, que realizou uma análise epidemiológica e exploratória da distribuição dos casos confirmados e óbitos por COVID-19 no estado do Piauí, região nordeste do Brasil. O referido estado possui 224 municípios, área territorial de 251.611,929 km², densidade demográfica de 12,40 hab./km², com população estimada para 2020 de 3.281.480 habitantes, (IBGE, 2021a).

As variáveis sociodemográficas (sexo, faixa etária) e variáveis epidemiológicas (número de casos confirmados e número de óbitos por município de residência, e frequência de comorbidades ou fatores de risco dentre os óbitos) foram obtidas a partir de dados secundários, provenientes das notificações do Sistema de Vigilância de Síndromes Respiratórias Agudas, disponibilizados no painel epidemiológico COVID-19, da Secretária de Saúde do Estado do Piauí (SESAPI, 2021).

Foram incluídos no estudo os casos confirmados e os óbitos por COVID-19, que foram notificados no ano de 2020, no estado do Piauí. Os indivíduos com teste negativo para a infecção ou óbitos suspeitos que não tiveram a confirmação do diagnóstico da doença ou que não foram notificados até a data de finalização da coleta não foram incluídos no estudo.

Para caracterização epidemiológica, foram realizadas estatísticas descritivas. $\mathrm{Na}$ análise espacial, as taxas de incidência e de mortalidade por município do estado, foram utilizadas para calcular o Índice de Moran Global, que constatou autocorrelação espacial positiva e em sequência foi avaliada a autocorrelação local, através do LISA (Local Indicators of Spatial Association). Para a representação espacial do diagrama de espalhamento de Moran, foram utilizados os Mapas de Moran (Moran Maps) que consideram o mapeamento dos municípios com diferenças estatisticamente significativas ( $\mathrm{p}<0,05)$, através do software Quantum Geographic Information System (QGIS) versão 2.14.8. Neste estudo, os cartogramas exibiram o delineamento de aglomerados espaciais em quatro tipos:

"Hotspots" (Alto-alto): áreas formadas por municípios com altas taxas de incidência e circundadas por municípios de altas taxas de incidência;

"Coldspots" (Baixo-baixo): áreas formadas por municípios com baixas taxas de incidência e circundadas por municípios com baixas taxas de incidência;

"Outliers" (Alto-baixo): áreas formadas por municípios com altas taxas da incidência e circundadas por municípios de baixas incidências;

"Outliers" (Baixo-alto): áreas formadas por municípios com baixas taxas de incidências e circundadas por municípios de altas taxas de incidências.

Os dados populacionais e vetoriais dos mapas da divisão dos limites municipais do estado do Piauí, foram obtidos a partir do banco de dados do Instituto Brasileiro de Geografia e Estatística (IBGE, 2021b; 2021c).

\section{Resultados e Discussão}

Até 31 de dezembro de 2020, o Brasil notificou 7.675.973 casos confirmados e 194.949 óbitos por COVID-19 (Brasil, 2020). No Piaú́, o primeiro caso confirmado e o primeiro óbito ocorreram nos dias 19 de março de 2020 e 27 de março de 2020, respectivamente. Até o dia 31 de dezembro de 2020, o Piauí possuía 143.179 casos confirmados da doença com registros em todos os municípios. No período ocorreram 2.840 mortes e apenas 24 municípios não tinham registros de óbitos de pessoas 
residentes.

Do total de casos confirmados, observa-se na Tabela 1, que houve predomínio em indivíduos do sexo feminino (54,6\%), enquanto os óbitos foram mais frequentes no sexo masculino (58,5\%). O maior número de casos ocorreu em adultos de 30-39 anos, intervalo que representa grande parte da população economicamente ativa, enquanto os óbitos ocorreram mais em idosos.

Tabela 1: Caracterização dos casos confirmados e óbitos por COVID-19, no estado do Piauí, no ano de 2020, segundo sexo e faixa etária. Piauí/Brasil, 2020.

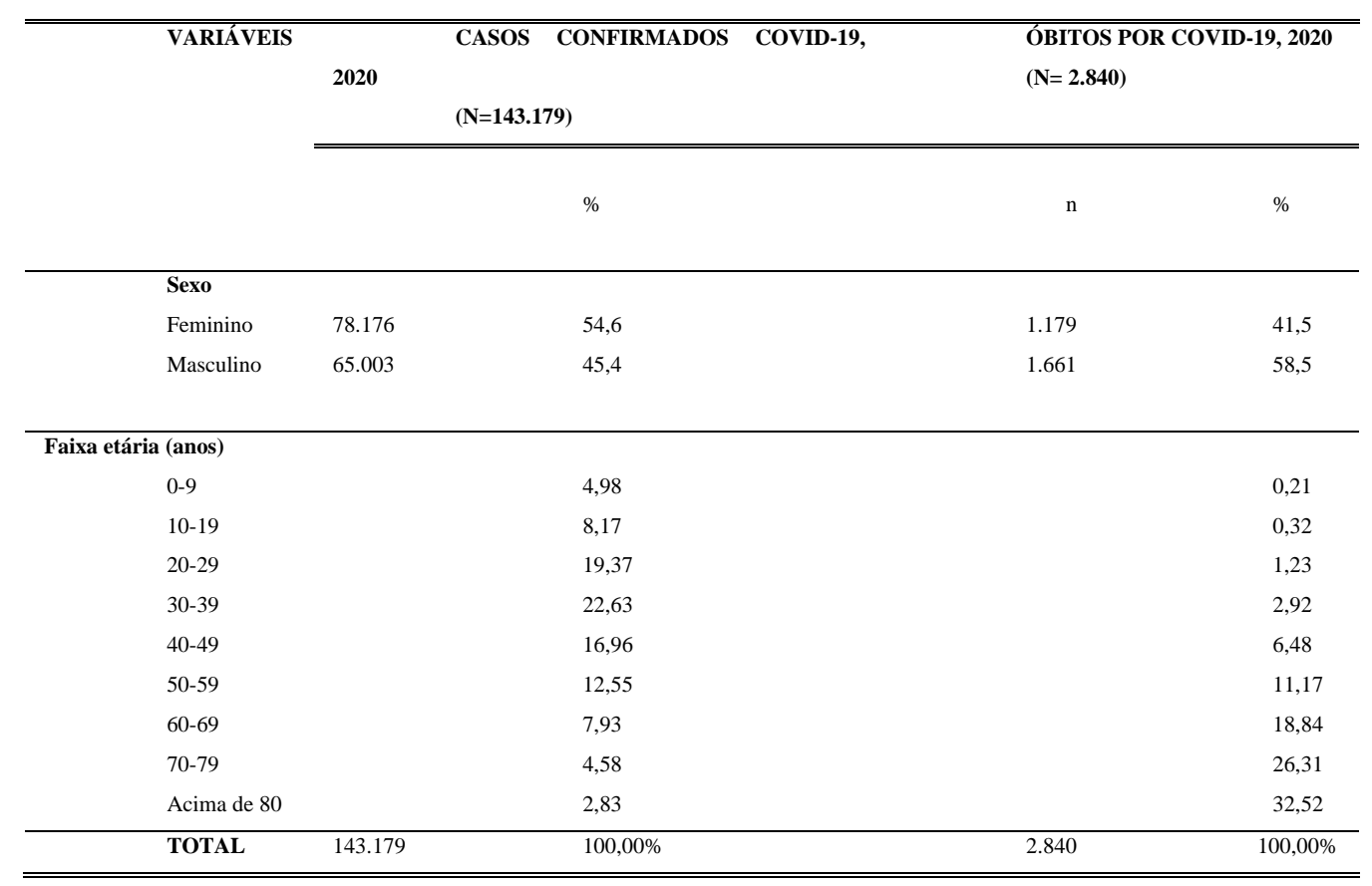

Legenda: N= número; \% porcentagem. Fonte: Elaboração própria, dados da SESAPI (2020).

Dos óbitos que ocorreram por COVID-19, apenas 6,06\% dos casos não apresentaram comorbidades ou fatores de risco associados. As comorbidades mais evidenciadas dentre os óbitos, foram cardiopatias incluindo hipertensão $(45,51 \%)$ e diabetes $(25,26 \%)$.

Esses dados apresentam similaridade com outros estudos, que verificaram que o COVID-19 apresentou maior número de casos em indivíduos do sexo feminino, adultos jovens (Machado et al., 2021; de Brito \& de Oliveira et al., 2021; dos Santos et al., 2021) e causou mais mortes entre idosos, do sexo masculino, com doenças crônicas (Silva et al., 2020; Machado et al., 2021; de Brito \& de Oliveira et al., 2021).

Cabe salientar que a Organização Mundial de Saúde (OMS, 2020), preconiza que indivíduos acima de 60 anos encontram-se no grupo de risco, particularmente devido às alterações naturais que ocorrem no sistema imunológico com a idade, favorecendo a agressividade da infecção.

Na análise espacial os 143.179 casos confirmados, que ocorreram no Piauí, no ano de 2020, foram georreferenciados. A frequência em número absoluto e a incidência são apresentados na Figura 1. 
Figura 1: Distribuição dos casos confirmados de COVID-19, no estado do Piauí, no ano de 2020 (N=143.179). Piauí/ Brasil, 2020 .

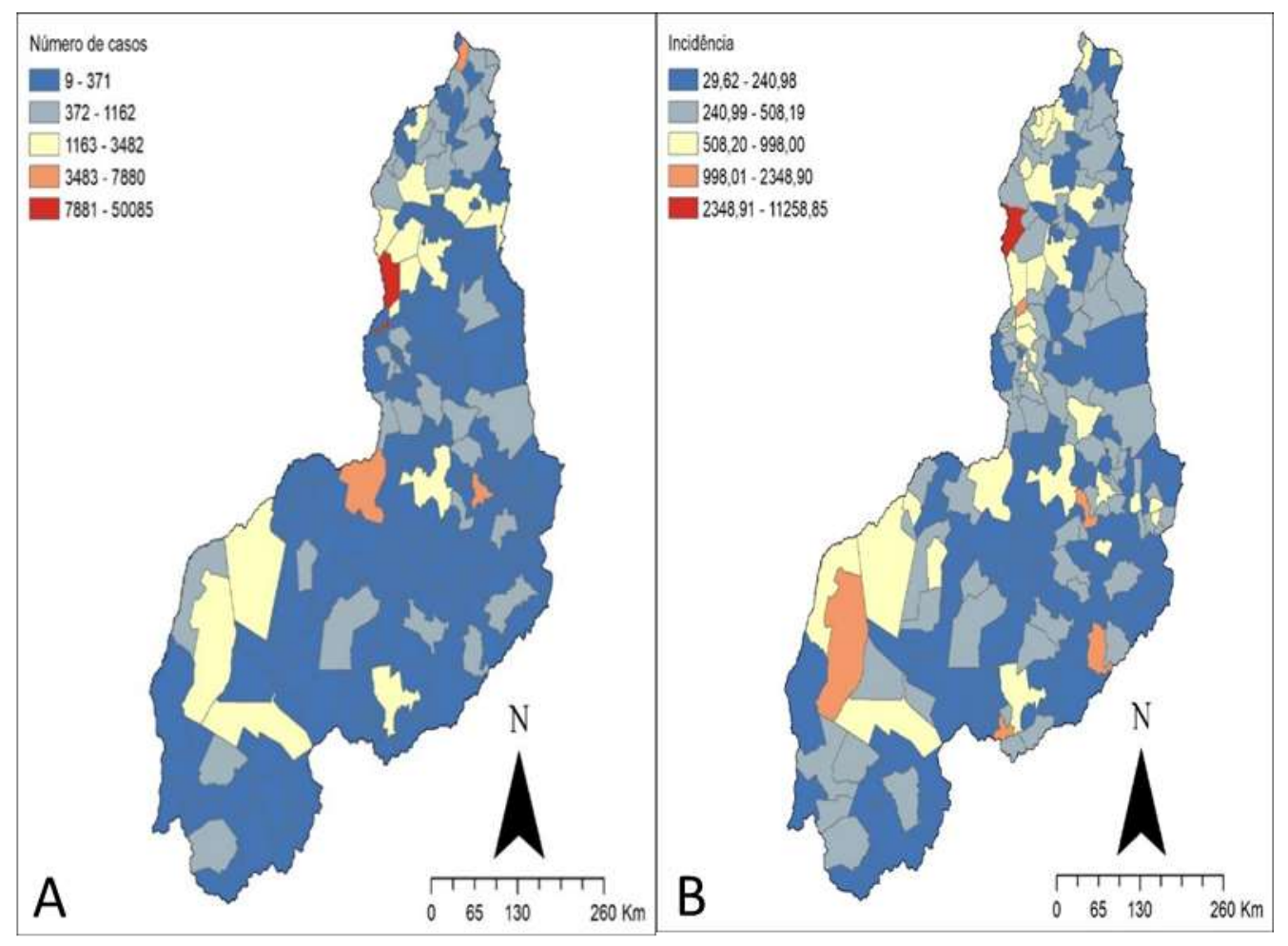

Legenda: 1A) Frequência absoluta dos casos; 1B) Incidência. Fonte: Autores.

Observa-se, na Figura 1A, que o estado do Piauí apresenta casos confirmados de COVID- 19 em todos os municípios. A capital Teresina apresentou maior número, tendo 50.085 casos no período. Vale ressaltar que Teresina é o município mais populoso do Piauí com população estimada, em 2020, de 868.075 mil habitantes (IBGE, 2021a). Em seguida, as cidades Parnaíba, Picos e Floriano apresentaram 7.880, 4.937 e 4.265 casos confirmados da doença, respectivamente, e apresentaramse na faixa de 3.483 a 7.880 mil casos, ocupando a segunda faixa com maior número absoluto. Ressalta-se que estes municípios estão entre os mais populosos do Piauí.

No contexto da atual pandemia do novo coronavírus, diversas investigações assinalam que a COVID-19 dissemina-se de maneira acelerada em grandes cidades (Stier et al., 2020). Esses municípios de maior porte populacional concentram os principais equipamentos, infraestrutura e serviços no Piauí, destacando-se aqueles destinados à saúde, como hospitais de maior complexidade clínica e com maior capacidade para receber pacientes. Essas cidades, devido à falta de hospitais de complexidade no interior do Estado, são referências para o tratamento de várias doenças, como a COVID-19.

Entretanto, quando se considera os coeficientes de incidência de COVID-19, o maior coeficiente do estado foi registrado no município de União (11.258,85/100 mil hab.), seguido dos municípios de Várzea Branca (2.348,90/100 mil hab.), Santa Cruz do Piauí (1.160,26/ 100 mil hab.), Baixa Grande do Ribeiro (1.354,22/100 mil hab.), Lagoa do Barro do Piauí (1.080,56/100 mil hab.) e Demerval Lobão (1.074,04/100 mil hab.) (Figura 1B). Dessas cidades, apenas União apresenta população superior a 15.000 mil habitantes e com exceção desta, as outras são cidades providas de poucos recursos na saúde.

A taxa de incidência de COVID-19 no estado do Piauí foi de 4.363,24/100 mil hab. A medida dessa frequência verificada no estado foi superior à média nacional (3.652,7/100 mil hab.) (Brasil, 2021).

A correlação espacial calculada por meio do índice de Moran Global evidenciou a dependência espacial entre os 
coeficientes de incidência dos municípios do estado do Piauí. Aplicando o método LISA, observou-se autocorrelação local e aglomerados espaciais (Figura 2).

Figura 2: Autocorrelação local da incidência dos casos confirmados de COVID-19, no estado do Piauí, no ano de 2020 (N=143.179). Piauí/ Brasil, 2020.

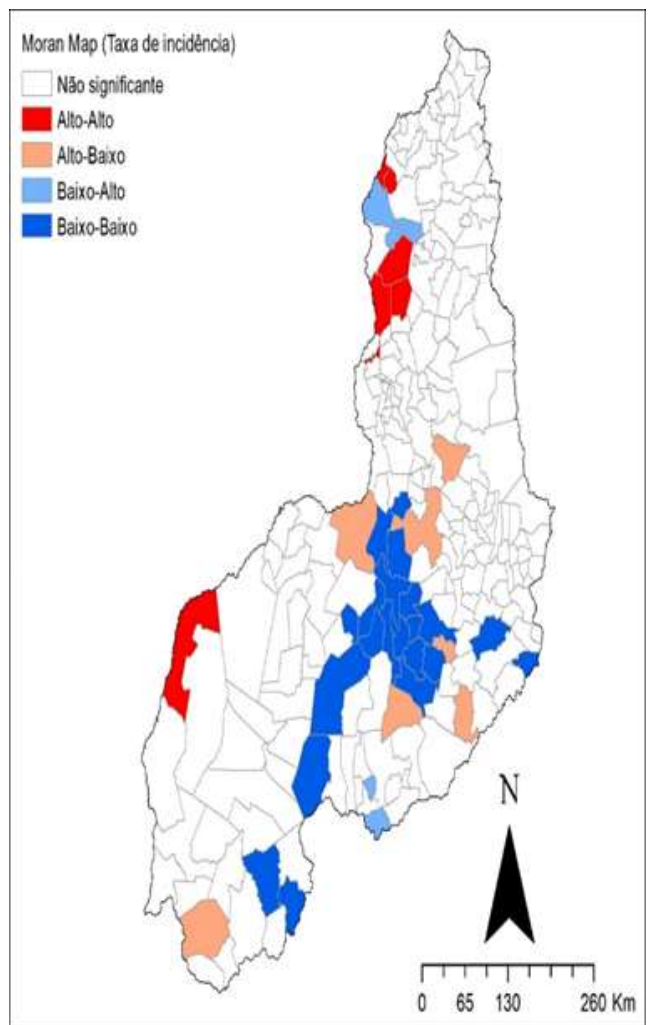

Fonte: Autores

Na Figura 2 é possível identificar a formação de clusters (Alto-alto) nos municípios de Teresina, José de Freitas, Altos, Porto, Nossa Senhora dos Remédios, Ribeiro Gonçalves e seus vizinhos. Esses clusters identificados possuem limites com cidades do estado do Maranhão. Os municípios de Miguel Alves, Lagoa alegre, cabeceiras do Piauí, Fartura do Piauí e Bomfim constituem os “Outliers” Baixo-alto. Já os “Outliers” Alto-baixo, correspondem aos municípios de Oeiras, Valença do Piauí, Floriano, Lagoa do Barro do Piauí, Bela Vista do Piauí, João Costa e Corrente. É possível observar que o Piauí ainda apresenta áreas com padrão Baixo-Baixo, que devem ser protegidos para evitar a introdução de casos nesses locais.

A taxa de mortalidade por COVID-19 do estado do Piauí que foi calculada em 73,92/100 mil hab. O número de óbitos e a mortalidade por município estão dispostos no mapa da Figura 3. 
Figura 3: Distribuição dos óbitos por COVID-19, no estado do Piauí, no ano de 2020 (N=2.840). Piauí/ Brasil, 2020.

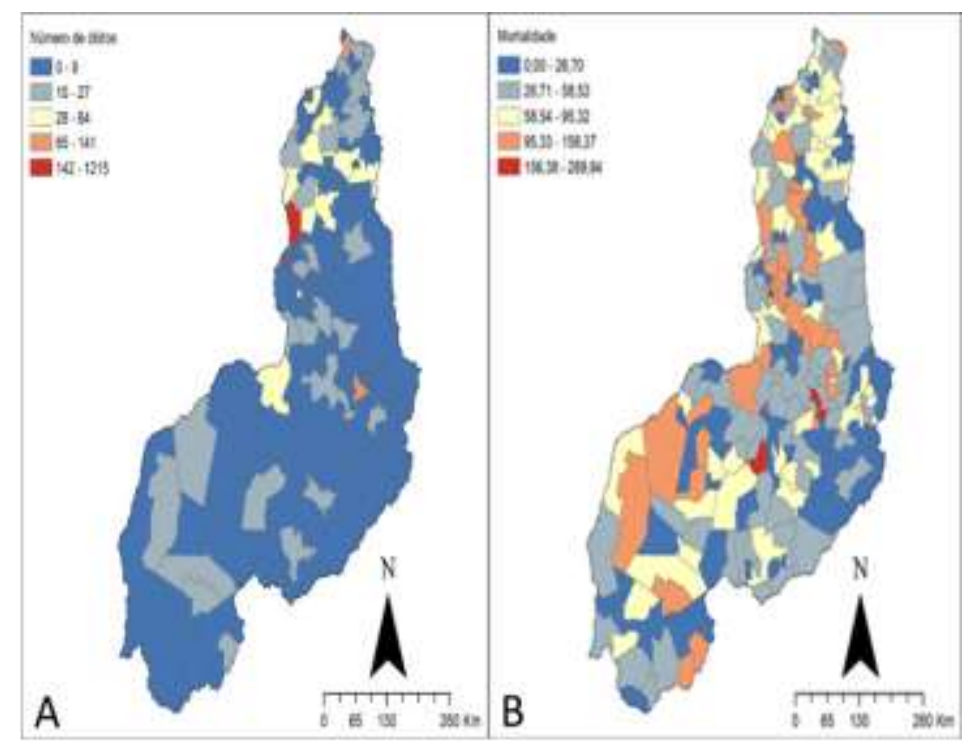

Legenda:3A) Frequência absoluta de óbitos; 3B) Mortalidade. Fonte: Autores.

Observa-se através da Figura que 3A que a capital Teresina apresentou o maior número de óbitos (1.215), seguido pelos municípios de Parnaíba (141) e Picos (96), sendo estes os municípios mais populosos do Piauí.

A maior taxa de mortalidade encontrada no estado foi a do município de Água Branca (269,94/100 mil hab.), seguindo-se Pajeú do Piauí (206,55/100 mil hab.) e Santa Cruz do Piauí (192,31/100 mil hab.).

O índice de Moran Global demonstrou a existência de autocorrelação espacial, evidenciando a dependência espacial entre os coeficientes de mortalidade dos municípios piauienses. A Figura 4 apresenta os aglomerados espaciais nos municípios, identificados de acordo com o índice de Moran local.

Figura 4: Autocorrelação local da mortalidade da COVID-19, no estado do Piauí, no ano de 2020 (N: 2.840). Piauí/ Brasil, 2020 .

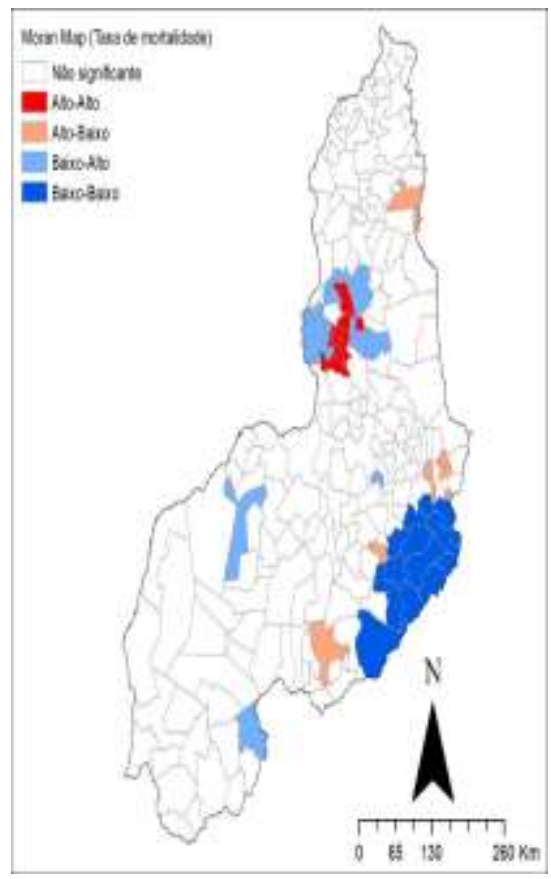

Fonte: Autores. 
Foi possível verificar formação de clusters Alto-alto para mortalidade nos municípios de Água Branca, Angical do Piauí, Barro Duro, Beneditinos, Hugo Napoleão, Jardim do Mulato, Lagoinha do Piauí, Olho D'água do Piauí, Passagem Franca do Piauí, Santo Antônio dos Milagres, municípios estes situados na Região do Entre Rios e Prata Do Piauí localizado na região Vale do Sambito. Alguns municípios que são vizinhos ao cluster citado e os municípios, Morro Cabeça no Tempo, Sebastião Leal e Wall Ferraz são "Outliers” Baixo-alto.

Observou-se formação de cluster Baixo-baixo entre municípios da região do semiárido do Piauí (Acauã, Betânia do Piauí, Campo Alegre do Fidalgo, Conceição do Canindé, Caridade, Curral Novo do Piauí, Dom Inocêncio, Jacobina do Piauí, Lagoa do Barro do Piauí, Massapê do Piauí, Patos do Piauí, Paulistana, Queimada Nova, São Francisco de Assis do Piauí, Simões). Nessa região foi possível verificar a maioria dos municípios que constituem os "Outliers" Alto-baixo.

\section{Conclusão}

A COVID-19, no estado do Piauí, no ano de 2020, apresentou maior número de casos em indivíduos do sexo feminino, adultos jovens e causou mais mortes entre idosos, do sexo masculino, com doenças crônicas. A capital Teresinense liderou o número de casos e de óbitos pela doença devido, provavelmente, à alta densidade populacional. Entretanto, quando se considera os coeficientes de incidência e mortalidade de COVID-19, as maiores taxas do estado foram registradas nos municípios de União e Água Branca, respectivamente.

As análises realizadas proporcionaram a visualização de aglomerados espaciais e a identificação de áreas vulneráveis, fornecendo informações que não seriam visualizadas trabalhando apenas com dados tabulares. Os resultados sugerem a necessidade da intensificação de estratégias de controle, como o distanciamento social, para que a velocidade de transmissão do COVID-19 seja reduzida e, consequentemente, se evite o aumento dos óbitos. Devido à alta transmissibilidade, supõe-se que essa distribuição altera-se com o tempo, fazendo-se necessários estudos contínuos que empreguem essas análises, contribuindo para compreensão da evolução da COVID-19 no estado do Piauí.

Algumas limitações, como a definição de caso confirmado que está fundamentada em critério laboratorial, baixa testagem da população e existência de pessoas assintomáticas portadora do vírus, podem levar a subnotificação e consequentemente subestimativa dos dados. A ausência de pesquisas semelhantes no estado limita a comparabilidade.

Ressalta-se a importância da análise espacial como alternativa metodológica para auxiliar desde o planejamento até a avaliação das ações em saúde.

\section{Agradecimentos}

Os autores agradecem o apoio da Coordenação de Aperfeiçoamento de Pessoal de Nível Superior (CAPES).

\section{Referências}

Belforte, L. C. M., Reis, R. D. S. P., da Silva, G. P., \& de Aguiar Cavalcante, M. M. (2020). Leitura geográfica no contexto da covid-19 em Rondônia ao norte do Brasil. Revista Tamoios, 16(1).

Cardoso, P. V., da Silva Seabra, V., Bastos, I. B., \& Costa, E. D. C. P. (2020). A importância da análise espacial para tomada de decisão: um olhar sobre a pandemia de COVID-19. Revista Tamoios, 16(1).

Channappanavar, R., Zhao, J., \& Perlman, S. (2014). T cell-mediated immune response to respiratory coronaviruses. Immunologic research, 59(1), 118-128.

Cheng, V. C., Lau, S. K., Woo, P. C., \& Yuen, K. Y. (2007). Severe acute respiratory syndrome coronavirus as an agent of emerging and reemerging infection. Clinical microbiology reviews, 20(4), 660-694.

Cheng, Z. J., \& Shan, J. (2020). 2019 Novel coronavirus: where we are and what we know. Infection, 48(2), $155-163$.

de Brito, I. C., \& de Oliveira, A. S. (2021). Perfil epidemiológico da covid-19 no estado da bahia. perfil epidemiológico da covid-19 no estado da bahia, 1388. 
dos Santos Bezerra, B., da Silva, S. R. M., \& Araújo, T. P. (2021). Evolução dos casos de COVID-19 de dezembro de 2019 a outubro de 2020 no Maranhão. Saúde Coletiva (Barueri), 11(63), 5536-5545.

dos Santos, F. D. A., \& Mendes, L. M. S. (2020). Análise bivariada, multivariada e espacial da ocorrência do sars-cov-2 nos municípios do estado do Piauí, nordeste do brasil. Hygeia-Revista Brasileira de Geografia Médica e da Saúde, 16, 249-263.

Gorbalenya, A. E., Baker, S. C., Baric, R., Groot, R. J. D., Drosten, C., Gulyaeva, A. A., ... \& Ziebuhr, J. (2020). Severe acute respiratory syndrome-related coronavirus: The species and its viruses-a statement of the Coronavirus Study Group.

Heymann, D. L., \& Shindo, N. (2020). COVID-19: what is next for public health?. The Lancet, 395(10224), 542-545.

Huang, C., Wang, Y., Li, X., Ren, L., Zhao, J., Hu, Y., ... \& Cao, B. (2020). Clinical features of patients infected with 2019 novel coronavirus in Wuhan, China. The lancet, 395(10223), 497-506.

Instituto Brasileiro de Geografia e Estatística. Censo 2010. <https://censo2010.ibge.gov.br/resultados.html>.Acesso em: 04 jan. 2021 b.

Instituto Brasileiro de Geografia e Estatística. Malha municipal digital do Brasil: situaçãoem $2018 . \quad$ Rio de Janeiro: IBGE, 2018. <ftp://geoftp.ibge.gov.br/malhas_digitais/>.

Instituto Brasileiro de Geografia e Estatística. Piauí. 〈https://cidades.ibge.gov.br/brasil/pi/historico〉.

Kampf, G., Todt, D., Pfaender, S., \& Steinmann, E. (2020). Persistence of coronaviruses on inanimate surfaces and their inactivation with biocidal agents. Journal of hospital infection, 104(3), 246-251.

Kang, D., Choi, H., Kim, J. H., \& Choi, J. (2020). Spatial epidemic dynamics of the COVID-19 outbreak in China. International Journal of Infectious Diseases, 94, 96-102.

Lai, C. C., Shih, T. P., Ko, W. C., Tang, H. J., \& Hsueh, P. R. (2020). Severe acute respiratory syndrome coronavirus 2 (SARS-CoV-2) and coronavirus disease-2019 (COVID-19): The epidemic and the challenges. International journal of antimicrobial agents, 55(3), 105924

Lipsitch, M., Swerdlow, D. L., \& Finelli, L. (2020). Defining the epidemiology of Covid-19—studies needed. New England journal of medicine, 382(13), $1194-1196$

Machado, A. G., dos Santos Batista, M., \& de Souza, M. C. (2021). Características epidemiológicas da contaminação por COVID-19 no estado da Bahia. Revista Enfermagem Contemporânea, 10(1).

Organização Pan-Americana da Saúde. Folha informativa - COVID-19 (doença causada pelo novo coronavírus) [Internet]. Brasília: Organização PanAmericana da Saúde; 2021a [citado 2021 Jan 01]. https://www.paho.org/bra/index.php?option=com_content\&view=article\&id=6101:covid19\&Itemid=875

Secretaria de Estado da Saúde do Piauí. Painel Epidemiológico Covid-19: Piauí. <https://datastudio.google.com/reporting/a6dc07e9-4161-4b5a-9f2a6f9be486e8f9/page/2itOB>.

Silva, T. M. da, Silva, L. E. A., Silva, A. R. da, Cavalcante, L. C. da S., Costa, S. de J., Silva, R. P. da, Moura, M. C. L. de, ... \& Santos, J. C. (2020). Perfil epidemiológico da morbimortalidade da Covid-19 no Estado do Piauí: uma atualização do cenário atual. Research, Society and Development, 9(8), e803986091.

Stier, A., Berman, M., \& Bettencourt, L. (2020). COVID-19 attack rate increases with city size. Mansueto Institute for Urban Innovation Research Paper Forthcoming.

Thompson, R. N. (2020). Novel coronavirus outbreak in Wuhan, China, 2020: intense surveillance is vital for preventing sustained transmission in new locations. Journal of clinical medicine, 9(2), 498.

Thompson, R. N. (2020). Novel coronavirus outbreak in Wuhan, China, 2020: intense surveillance is vital for preventing sustained transmission in new locations. Journal of clinical medicine, $9(2), 498$.

Van Doremalen, N., Bushmaker, T., Morris, D. H., Holbrook, M. G., Gamble, A., Williamson, B. N., ... \& Munster, V. J. (2020). Aerosol and surface stability of SARS-CoV-2 as compared with SARS-CoV-1. New England journal of medicine, 382(16), 1564-1567.

World Health Organization. Situation report-81: coronavirus disease 2019 (COVID-19) [Internet]. Geneva: World Health Organization; $2021 \mathrm{~b}$ [cited 2021 Jan 01]. https://www.who.int/emergencies/diseases/novel-coronavirus-2019/situation-reports

Zhu, N., Zhang, D., Wang, W., Li, X., Yang, B., Song, J., ... \& Tan, W. (2020). A novel coronavirus from patients with pneumonia in China, 2019. New England journal of medicine.

Zumla, A., Chan, J. F., Azhar, E. I., Hui, D. S., \& Yuen, K. Y. (2016). Coronaviruses- drug discovery and therapeutic options. Nature reviews Drug discovery, 15(5), 327-347. 